\title{
LE GRAPHÈNE : \\ à nos crayons pour redessiner le paysage de l'optoélectronique
}

\author{
Thomas WOOD \\ Malik KEMICHE \\ Jérémy LHUILLIER \\ Ségolène CALLARD \\ Christelle MONAT* \\ Institut des Nanotechnologies \\ de Lyon (INL, UMR-CNRS 5270), \\ École Centrale de Lyon, \\ Université de Lyon, Écully, France \\ *christelle.monat@ec-lyon.fr
}

\begin{abstract}
Après avoir suscité l'intérêt du secteur microélectronique, le graphène, matériau bidimensionnel constitué d'un monofeuillet d'atomes de carbone, pourrait bien devenir un matériau phare de l'optoélectronique. Ses propriétés optiques le rendent pertinent pour un large spectre d'applications, dont le potentiel est loin d'être épuisé, en particulier pour l'optique intégrée. Renforcer son interaction avec la lumière reste un enjeu important pour la miniaturisation des composants.
\end{abstract}

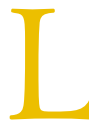

es propriétés remarquables du graphène, en particulier son absorption universelle, sa réponse optique accordable, ou encore la haute mobilité des porteurs et leur relaxation rapide, le prêtent à une myriade d'applications en optoélectronique incluant la photodétection, les biocapteurs, les cellules solaires, ou la modulation et le routage de signaux lumineux (figure 1) $[1,2]$. Les propriétés optiques nonlinéaires du graphène sont également exploitées pour générer des impulsions lumineuses ou laissent envisager des fonctions à commande optique [3]. Surtout, ce matériau promet la réalisation de composants optoélectroniques fonctionnant rapidement, bien au-delà de la dizaine de $\mathrm{GHz}$, et couvrant une bande spectrale étendue, du visible au moyen-IR. Son intégration relativement aisée dans des dispositifs fibrés ou planaires lui donne par ailleurs un atout considérable en termes de réduction des coûts

\section{Méthodes de synthèse de graphène : vers une production de masse?}

Depuis l'exfoliation mécanique qui a permis, en 2004, d'isoler une monocouche de carbone à partir d'adhésif et de graphite, les techniques de production de graphène se sont multipliées, cherchant un compromis entre surface, qualité et coût [1]. Si le graphène ainsi exfolié présente une bonne qualité, sa surface est réduite $\left(<1 \mathrm{~mm}^{2}\right)$. L'exfoliation en voie liquide permet d'obtenir un mélange de flocons de graphène en solution qui peuvent être dispersés sur un substrat quelconque, mais le taux de couverture final reste faible. Une voie alternative est la synthèse de graphène par dépôt en phase vapeur (CVD) sur substrat métallique de cuivre ou nickel. Cette technique mature est utilisée par les industries qui commercialisent aujourd'hui le graphène, et permet le report de grandes surfaces $\left(\approx 1 \mathrm{~cm}^{2}\right)$ sur tout type de substrat pour la photonique. Néanmoins, les impuretés et défauts introduits lors du transfert tendent à dégrader ses performances électroniques. L'épitaxie directe de graphène sur le substrat d'intérêt (par exemple sur $\mathrm{SiC}$ ) permet de s'affranchir de ces défauts mais reste difficile à généraliser. Notons les progrès réalisés sur substrat de SiGe ou sur nitrure de bore hexagonal ( $h-B N$ ), un autre matériau 2D, dont les propriétés complètent avantageusement celles du graphène. En effet, au-delà de la qualité du graphène, son interaction avec le substrat affecte grandement ses propriétés.

de fabrication. Cependant, l'épaisseur du graphène, limitée à quelques ångströms, reste un frein à la miniaturisation des composants et à leur intégration sur puce. Si son interaction avec la lumière est en effet large rapportée à son épaisseur, elle reste faible dans l'absolu. L'intégration du graphène dans des architectures optiques permettant de renforcer son interaction avec la lumière constitue un enjeu important pour créer des composants compacts et efficaces. Nous donnons ici un aperçu des applications exploitant ce matériau $2 \mathrm{D}$, illustré par quelques exemples de composants optoélectroniques à base de graphène.

\section{Le graphène pourr générer des impullsions laser}

L'absorption saturable reste la propriété optique du graphène la plus largement exploitée en termes d'applications. Combinée à un temps de relaxation court et un faible seuil de saturation, elle permet de générer des trains d'impulsions lumineuses ultra-rapides à partir de lasers dits à blocage de modes. Ces lasers impulsionnels émettent du visible au 
moyen infra-rouge pour le biomédical, le traitement des matériaux ou les communications optiques.

Depuis 2009, les lasers impulsionnels à base de graphène ont évolué pour délivrer des impulsions plus courtes, des puissances plus élevées et à un taux de répétition accru [3]. Le graphène est initialement exploité dans des lasers à fibre, où un à quelques dizaines de mono-feuillets sont insérés entre deux connecteurs [4] (figure 2a). Le taux de répétition est restreint à quelques $\mathrm{MHz}$ à cause de la longueur de cavité. Dans cette configuration, la puissance moyenne est souvent limitée à quelques $\mathrm{mW}$ par le seuil d'endommagement du graphène qui est traversé par l'intégralité du flux lumineux circulant dans la cavité fibrée. Dans des configurations en espace libre, quelques pourcents seulement du flux lumineux sont envoyés sur un substrat couvert de graphène, permettant de faire monter la puissance de sortie à plusieurs centaines de $\mathrm{mW}$. Ces lasers, très modulables, peuvent délivrer des impulsions dont la durée atteint $20 \mathrm{fs}$, en contrôlant la dispersion dans la cavité à l'aide de prismes, mais au détriment de la compacité et de la robustesse du dispositif. Pour augmenter la puissance des lasers fibrés, le graphène est intégré sur la périphérie de la fibre (figure $2 c, 2 d, 2 e$ ), compensant la réduction de l'interaction par unité de longueur du graphène avec le champ lumineux évanescent par une augmentation de la surface d'interaction. Des puissances de l'ordre du watt ont été obtenues dans des fibres à cristaux photoniques imprégnées de graphène.

\section{Propriétés optolélectroniques du graphène}

La structure de bandes électronique du graphène, monofeuillet de carbone à maille hexagonale (a), présente un cône de Dirac près d'un point de haute symétrie. Combinée à l'absence de gap à l'état intrinsèque (niveau de Fermi $E_{F}=0 \mathrm{eV}$ ), cette dispersion linéaire confere au graphène une absorption indépendante de l'énergie des photons incidents du visible au moyen IR (b). L'ajustement du niveau de Fermi par dopage (en statique) ou par le biais d'une tension de contrôle (en dynamique) permet de contourner partiellement cette absence de gap et d'accorder les propriétés optiques du graphène. Par exemple, ce matériau peut être rendu tour à tour transparent ou opaque selon que l'énergie de photons est inférieure ou supérieure à $2 \mathrm{E}_{\mathrm{F}}$, permettant ainsi la réalisation de fonctions accordables électriquement avec, en principe, de faibles tensions de contrôle. De manière générale, toutes les propriétés du graphène dépendent fortement de $E_{F}$. Il possède une absorption saturable : en présence de fortes intensités lumineuses, le remplissage de la bande de conduction bloque les transitions interbandes, rendant le matériau transparent (c). La structure planaire du graphène conduit également à une réponse anisotrope. Enfin la contribution des transitions intrabandes et interbandes dominent tour à tour le comportement optique selon l'énergie des photons $(\mathrm{d})$, donnant naturellement accès à des régimes plasmoniques du THz au moyen IR.

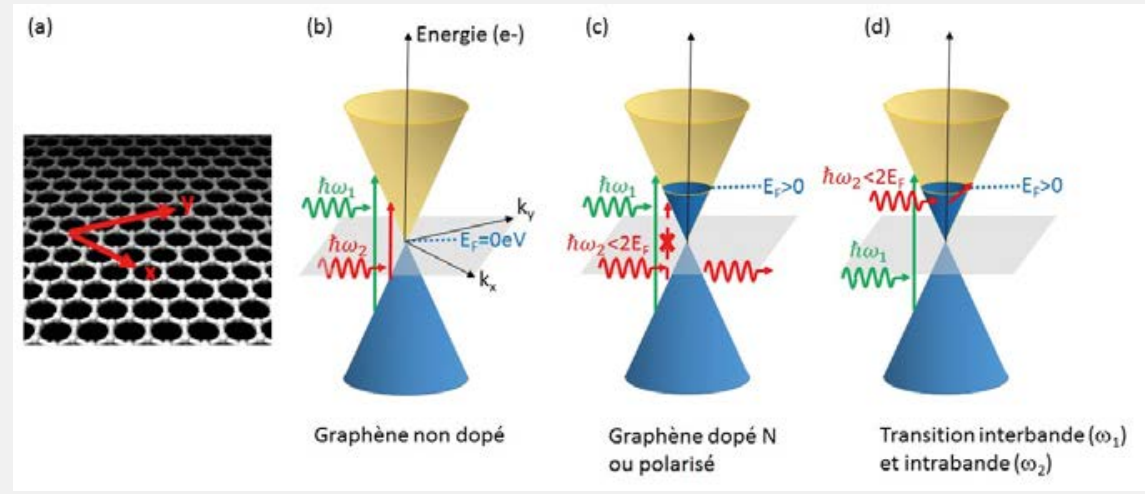

Figure 1. Propriétés optolélectroniques du graphène et applications.
D'autres applications, comme les télécommunications optiques, visent plutôt des taux de répétition élevés émis par des dispositifs compacts et intégrés sur puce. Des lasers à guide d'onde inscrits sur verre dopé ou des lasers fibrés à cavité linéique (figure 2f), dans lesquels le graphène recouvre l'un des miroirs, se rapprochent le plus de ce cahier des charges, avec des dimensions centimétriques et des taux de répétition de l'ordre de la dizaine de $\mathrm{GHz}$ [5]. La réalisation de lasers pulsés intégrés sur puce reste un véritable défi. De nouvelles architectures nanophotoniques permettront peut-être de franchir cette nouvelle barrière et constituent l'un des objectifs du projet européen GRAPHICS à l'INL [6].

\section{Optoélectironique à base de graphène: un nouveau paysage de composants}

Le potentiel du graphène pour l'optoélectronique ne peut être mieux valorisé qu'en recensant la diversité des composants réalisés et réalisables (figure 3). Extrêmement versatile, le graphène sert tantôt d'électrode transparente pour le photovoltaïque, en raison de la forte mobilité de ses électrons, tantôt d'absorbeur pour des photodétecteurs sensibles du visible à l'infra-rouge avec une large bande passante (plusieurs centaines de GHz en principe). Notons que ces réalisations sont jusqu'ici limitées à quelques dizaines de $\mathrm{GHz}$ par la constante RC du dispositif et la surface de détection réduite pénalise encore leur sensibilité.

La capacité surfacique réduite du graphène permet, à partir de faibles modifications de densités de charges, de changer radicalement ses propriétés optiques pour manipuler dynamiquement des signaux lumineux. On peut ainsi réaliser des modulateurs rapides $(>10 \mathrm{GHz})$ et peu gourmands en énergie. Ces composants, qui servent à encoder l'information sur un signal lumineux ou agissent comme interrupteurs optiques, sont 
a

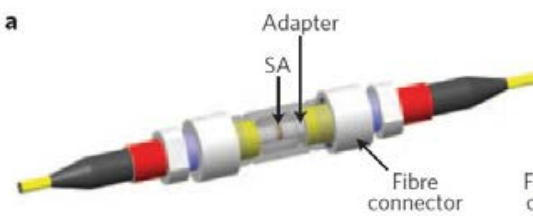

d

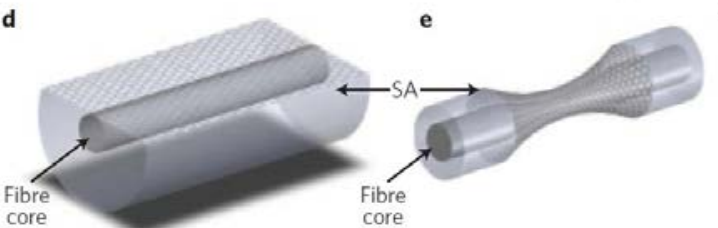

Figure 2. Intégration d'absorbant saturable à base de graphène pour des lasers impulsionnels à fibre.

Adapted by permission from Macmillan Publishers Ltd: Nature Photonics (A. Martinez and Z. Sun, Nanotube and graphene saturable absorbers for fibre lasers, Nature Photonics 7, 842-845 (2013), doi:10.1038/nphoton.2013.304), copyright (2013).

(a)
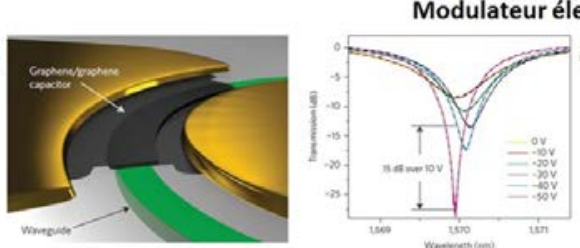

T. Phare et al., Nat. Photonics (2015) Interrupteur optique à commande électrique

(c)
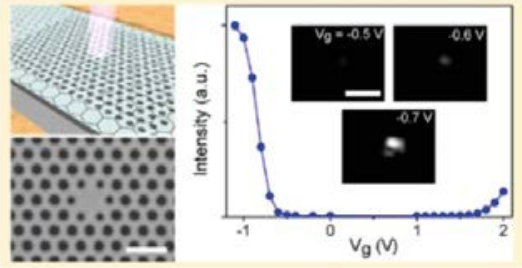

M. S. Hwang et al. Nanoletters 2017
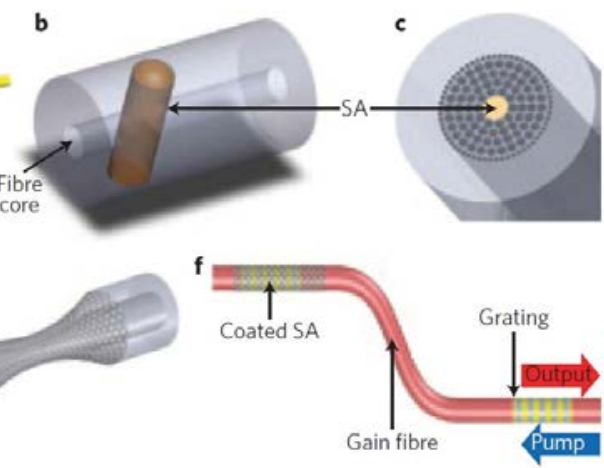
(d)

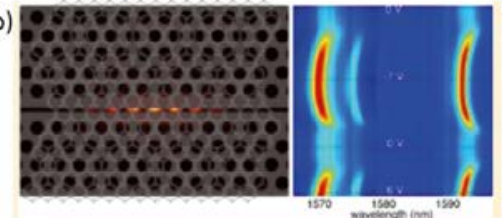

X. Gan et al. Nanoletters 2013

Polariseur contrôlé dynamiquement

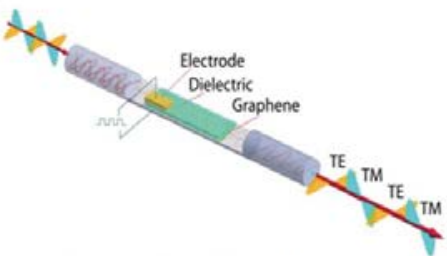

Q. Bao, ACS Nano 2012

Conversion de fréquences

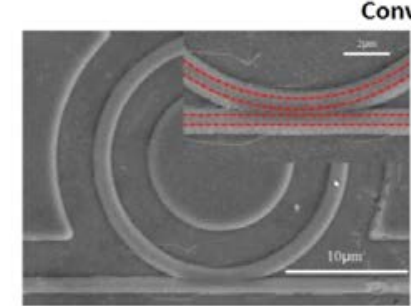

(e)

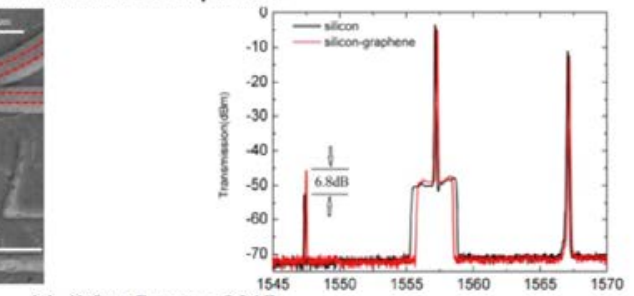

M. JiOpt Express 2015

\section{Obtenir le meilleur de l'innovation, c'est notre métier.}

Nos photomultiplicateurs en

silicium sont ultrasensibles,

petits, rapides et remplacent

efficacement les photomulti-

plicateurs classiques utilisant

un tube à électrons.

Figure 3. Quelques dispositifs optoélectroniques à base de graphène en optique guidée.

(a), (b) Modulateur électro-optique. (c) Interrupteur optique à commande électrique.

(d) Polariseur à contrôle dynamique. (e) Conversion de fréquences dans un micro-anneau hybride $\mathrm{Si}$ /graphène.

(a) Adapted by permission from Macmillan Publishers Ltd: Nature Photonics (T. Phare et al., Graphene electro-optic modulator with $30 \mathrm{GHz}$ bandwidth, Nature Photonics 9, 511-514 (2015), doi: 10.1038/ nphoton.2015.122), copyright (2015). (b) Reprinted with permission from D. Englund et al., High contrast electrooptic modulation of a photonic crystal nanocavity by electrical gating of graphene, Nanoletters 13, 691 (2013), doi: 10.1021/nl304357u. Copyright 2013 American Chemical Society. (c) Reprinted with permission from M.S. Hwang, Switching of photonic crystal lasers by graphene, Nanoletters (2017), doi: 10.1021/acs.nanolett.6b05207. Copyright 2017 American Chemical Society. (d) Reprinted with permission from Q. Bao, K.P. Loh, Graphene photonics, plasmonics, and broadband optoelectronic devices. ACS Nano 6, 3677-3694 (2012), doi:10.1021/nn300989g. Copyright 2012 American Chemical Society. (e) Reprinted with permission from Mengxi Ji et al., Enhanced parametric frequency conversion in a compact silicon-graphene microring resonator, Opt. Express 23, 1867918685 (2015), doi: 10.1364/OE.23.018679. Copyright 2015 The Optical Society.

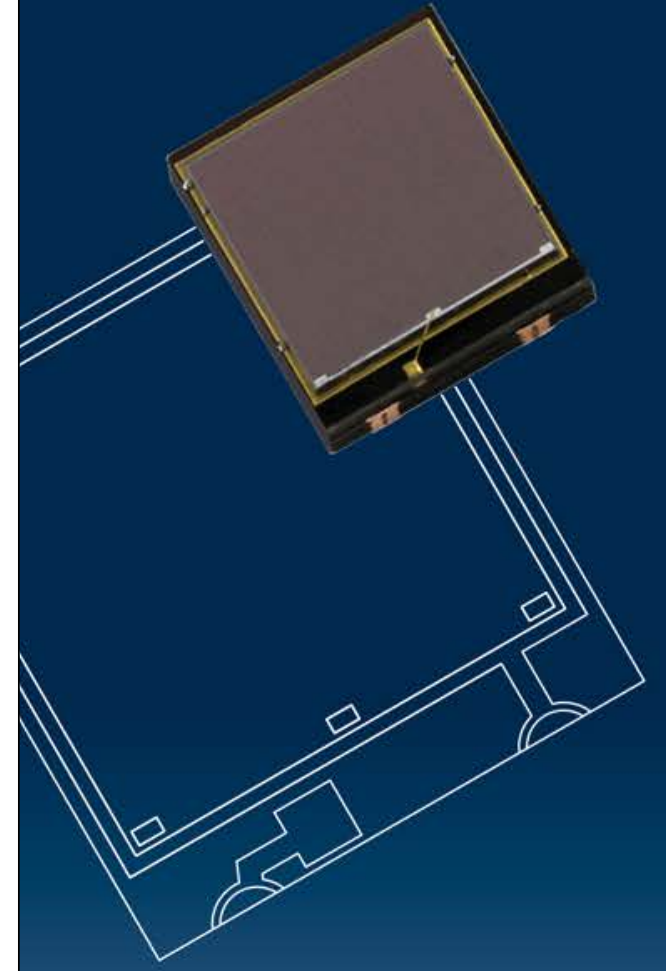


déclinés en version optique guidée (sur puce ou fibre) ou espace libre (graphène sur miroir) [7]. En appliquant une tension de contrôle, les indices réel et imaginaire du mode optique sont modifiés pour moduler la phase ou l'amplitude du signal optique en sortie. Quelque soit le scénario retenu, la réalisation d'un dispositif compact nécessite de renforcer l'interaction lumière/ graphène en plaçant judicieusement le graphène au cœur de guides diélectriques, ou de telle sorte qu'il interagisse fortement avec le champ évanescent des modes guidés. Citons, à titre d'exemple, un modulateur formé d'un micro-anneau de nitrure de silicium compact (diamètre $=80 \mu \mathrm{m})$ fonctionnant à $1,55 \mu \mathrm{m}$ et $30 \mathrm{GHz}$ avec une atténuation dynamique de $15 \mathrm{~dB}$, contrôlé par une tension variant sur $10 \mathrm{~V}$ [8]. Du côté des modulateurs en espace libre, le temps d'interaction de la lumière avec le graphène est augmenté via l'usage de structures résonantes périodiques (Bragg) ou plasmoniques, dont la fréquence de résonance est contrôlée électriquement. Les modulateurs évoluent vers des architectures plus compactes, plus rapides, avec des tensions de contrôle plus faibles, par exemple en utilisant des cristaux photoniques ou des empilements plus sophistiqués incluant plusieurs monocouches de graphène séparées par un diélectrique.

L'intégration du graphène sur fibre ou guide permet également de contrôler la polarisation d'un signal lumineux sur une large bande spectrale [9]. L'anisotropie optique du graphène conduit à un taux d'atténuation distinct pour les modes transverses TE et TM du guide hybride, qui permet un filtrage sélectif en polarisation $(\sim 27 \mathrm{~dB})$ après une distance de propagation de quelques millimètres. Contrairement à un film métallique, le choix de la polarisation de sortie peut être sélectionné dynamiquement par le biais d'une tension de grille appliquée au graphène.

Enfin, la réponse optique nonlinéaire du troisième ordre $\left(\chi^{(3)}\right)$ mesurée dans le graphène peut atteindre une valeur de plusieurs ordres de grandeur supérieure à celle du silicium, tempérée par une absorption qui reste à maîtriser. Cette réponse permet de manipuler optiquement les signaux lumineux, via des interrupteurs ultra-rapides à commande optique, mais aussi de convertir les photons en fréquence par génération d'harmoniques ou mélange à quatre ondes. En optique intégrée, les premières réalisations utilisent des guides hybrides en silicium dans lesquels le graphène interagit avec le champévanescent. Quelques démonstrations assez rares exploitent des cristaux photoniques ou micro-anneaux. La nonlinéarité effective est augmentée d'un facteur 10 environ par rapport au silicium seul, laissant une forte marge de progression.

\section{Evolution et enjeux pour la photonique intégrée: renforcer l'interaction lumière/graphène}

Pour exploiter tout le potentiel du graphène en optique intégrée, des stratégies sont déployées pour renforcer son interaction avec la lumière, par exemple, via l'usage de structures nanophotoniques à fort confinement optique. Ainsi, l'intégration de graphène sur des structures périodiques résonantes optimisées permet d'augmenter l'absorption intrinsèque d'une monocouche de graphène de $2,3 \%$ à $50 \%$ voire $100 \%$ en régime de couplage critique. Des géométries de guides à fentes ont également été proposées mais doivent tenir compte de l'anisotropie optique du graphène, qui interagit prioritairement avec les composantes de champ électrique situées dans le plan du feuillet.

Une autre alternative est l'exploitation des propriétés plasmoniques du graphène. Sa conductivité de surface permet l'existence de plasmons de surface associés à un mouvement collectif de charges. L'indice élevé de ces modes se traduit par une compression en longueur d'onde, attractive pour la miniaturisation des dispositifs optoélectroniques. Cette conductivité surfacique est de surcroît accordable, comme la fréquence de ces plasmons, par dopage ou contrôle électrique. Plusieurs études théoriques proposent ainsi leur utilisation pour du routage ou filtrage de signaux optiques à commande électrique. L'augmentation de la densité d'énergie lumineuse au niveau du feuillet de graphène induite par la localisation et compression spatiale de l'onde plasmonique sont également propices à renforcer les nonlinéarités du graphène.

Pour que le plasmon de surface $\mathrm{du}$ graphène existe aux longueurs d'onde télécom (1,3-1,6 $\mu \mathrm{m})$, un potentiel chimique élevé $(\approx 0,7 \mathrm{eV})$ difficile à atteindre est nécessaire. On peut s'en rapprocher en dopant

\begin{tabular}{|l|l|}
\hline PROPRIÉTÉS OPTIQUES DU GRAPHĖNE & COMPOSANTS OPTOÉLECTRONIQUES \\
\hline $\begin{array}{l}\text { Absorption saturable (rapide et large bande) } \\
\begin{array}{l}\text { Accordabilité électrique des propriétés optiques } \\
\text { (électro-absorption/ électro-réfraction) }\end{array}\end{array}$ & $\begin{array}{l}\rightarrow \text { Lasers pulsés } \\
\text { Modulateurs, interrupteurs optiques, } \\
\text { routage }\end{array}$ \\
\hline $\begin{array}{l}\text { Absorption (2,3\% par monocouche) du visible au moyen-IR } \\
\text { Nonlinéarité optique, réponse en } X^{(3)}\end{array}$ & $\rightarrow$ Photodétecteurs large bande \\
\hline $\begin{array}{l}\text { Anisotropie optique } \\
\text { Seuil de dommage optique élevé }\end{array}$ & $\rightarrow$ Conversion de fréquences, amplification, traitement tout optique de l'information \\
\hline
\end{tabular}


fortement le graphène de type $\mathrm{P}$, par l'intermédiaire de matériaux de revêtement avec un travail de sortie élevé (par exemple, $\mathrm{MoO}_{3}$ ) ou de traitements chimiques (oxydation par plasma $\mathrm{O}_{2}$ ). Par ailleurs, l'indice élevé des plasmons de surface du graphène rend leur excitation via l'espace libre ardue aux longueurs d'onde inférieures à quelques microns. Pour pallier cette difficulté, différentes approches ont été exploitées, fournissant in fine l'accord de phase nécessaire au couplage en espace libre. En nanostructurant le graphène pour former des nano-rubans ou des réseaux périodiques, les longueurs d'ondes des plasmons de surface excités dans le graphène ont pu être abaissées de $100 \mu \mathrm{m}$ à $15 \mu \mathrm{m}$ et $2 \mu \mathrm{m}$ entre 2013 et 2017 . Enfin, si l'excitation de plasmons de surface à 1,55 $\mu \mathrm{m}$ a été démontrée à l'aide de guides d'onde, les distances de propagation restent faibles (quelques centaines de nanomètres) en raison des pertes par absorption, un enjeu qui reste difficile à maîtriser.

\section{Conclusion}

Malgré la diversité de fonctions optoélectroniques démontrées en laboratoire, la richesse liée aux nombreuses propriétés optoélectroniques du graphène est loin d'avoir épuisé son potentiel applicatif. Les réalisations de composants ont succédé aux études spectroscopiques amont. Des structures toujours plus innovantes sont mises en œuvre pour renforcer l'interaction avec ce matériau 2D tandis que ses propriétés nonlinéaires sont encore assez peu exploitées pour la photonique intégrée sur puce - justement l'un des objectifs du projet H2020 GRAPHICS à l'INL. En parallèle, ce matériau $2 \mathrm{D}$ précurseur a inspiré des travaux sur ses cousins bidimensionnels avec des propriétés très complémentaires au graphène, par exemple h-BN isolant électrique ou $\mathrm{MoS}_{2}$ qui présente, en configuration $2 \mathrm{D}$, un gap direct donc un fort rendement de luminescence. Si le graphène reste actuellement le matériau bidimensionnel le plus mature déjà commercialisé, les hétérostructures 2D faites d'empilement de ces différents matériaux pourraient multiplier les opportunités pourlestechnologiesoptoélectroniques à venir [10].

Remerciements. Les auteurs tiennent à remercier le soutien de l'institut universitaire de France et du programme Européen 648546 ERC GRAPHICS «GRAphene nonlinear PHotonic Integrated CircuitS » financé par H2020.

\section{POUR EN SAVOIR PLUS}

[1] K.S. Novoselov et al., A roadmap for graphene, Nature (2012)

[2] F. Bonaccorso, Z. Sun, T. Hasan, A.C. Ferrari, Graphene photonics and optoelectronics, Nature Photonics (2010)

[3] X. Liu, Q. Guo, J. Qiu, Emerging low-dimensional materials for nonlinear optics and ultrafast photonics, Advanced Materials (2017)

[4] A. Martinez, Z. Sun, Nanotube and graphene saturable absorbers for fibre lasers, Nature Photonics (2013)

[5] D.P. Shepherd et al., Ultrafast high-repetition-rate waveguide lasers, IEEE J. Sel. Top. Quantum Electron. (2016)

[6] http://inl.cnrs.fr/graphics

[7] S. Yu, X. Wu, Y. Wang, X. Guo, L. Tong, 2D materials for optical modulation: challenges and opportunities, Advanced Materials (2017)

[8] T. Phare et al., Graphene electro-optic modulator with $30 \mathrm{GHz}$ bandwidth, Nature Photonics (2015).

[9] Q. Bao et al., Graphene photonics, plasmonics, and broadband optoelectronic devices, ACS Nano (2012)

[10] K.S. Novoselov, A. Mishchenko, A. Carvalho, A.H. Castro Neto, 2D materials and van der Waals heterostructures, Science (2016)

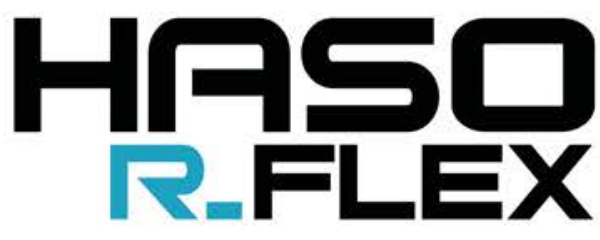

The most practical

and cost effective

metrology tool for

characterizing optical

components such as

large concave

mirrors, lenses,

complex optical

systems, and beam

expanders

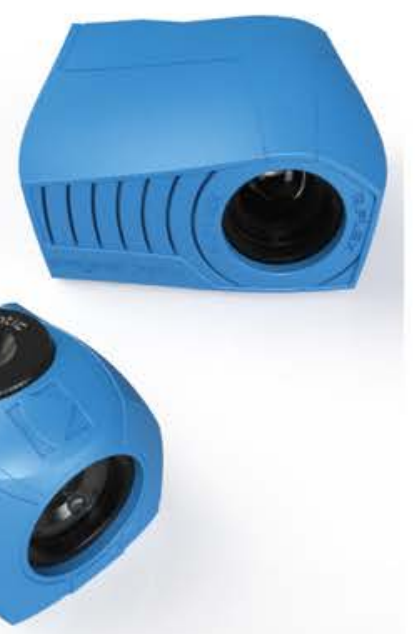

WAVELENGHT VISIBLE OR NIR

COMPACT

AND SELF-ILLUMINATION

METROLOGY TOOL

FOR LARGE OPTICS

EASYTO USE

AND VERSATILE

LARGE DYNAMIC

AND HIGH ACCURACY

Contact us for more details:

contact@imagine-optic.com

or +33164861560 